\title{
ORTHOGONALITY OF COSETS RELATIVE TO IRREDUCIBLE CHARACTERS OF FINITE GROUPS
}

\author{
RANDALL R. HOLMES
}

\begin{abstract}
Studied is an assumption on a group that ensures that no matter how the group is embedded in a symmetric group, the corresponding symmetrized tensor space has an orthogonal basis of standard (decomposable) symmetrized tensors.
\end{abstract}

\section{Introduction}

Let $V$ be a complex inner product space and let $G$ be a subgroup of the symmetric group $S_{n}$ for some $n$. Corresponding to an irreducible character of $G$ is a symmetrizer, a certain endomorphism of the $n$-fold tensor space $V^{\otimes n}$. The image under a symmetrizer of a standard basis vector of $V^{\otimes n}$ is called a standard (or decomposable) symmetrized tensor.

We seek conditions under which $V^{\otimes n}$ will have an orthogonal basis consisting entirely of standard symmetrized tensors (such a basis being called an o-basis for brevity). The problem of finding such conditions was first considered by Wang and Gong in [WG] where it was shown that if $G$ is the dihedral group of order eight (viewed naturally as a subgroup of $S_{4}$ ), then $V^{\otimes 4}$ has an o-basis. In subsequent work [HT], Tam and the author showed that more generally if $G$ is a dihedral group of order a power of two, then the corresponding tensor space has on o-basis. Moreover, it was noted there that this fact is independent of the particular embedding of the dihedral group inside the symmetric group. So, for instance, an o-basis exists for $V^{\otimes n}$ where $n$ is the order of the dihedral group (still assumed to be a power of two) and the embedding is the one given by Cayley's Theorem.

Motivated by this example, we give in this paper conditions on a finite group ensuring that, regardless of how it is embedded in a symmetric group, the corresponding tensor space will have an o-basis. We call a group satisfying this condition an o-basis group.

In Section 1, we state the definition of an o-basis group and establish some properties. In Section 2 , we review more carefully the notion of an o-basis of a tensor space and then give connections between this notion and that of an o-basis group. Finally, we show in Section 3 that the class of o-basis groups contains some interesting groups-the extra special $p$-groups ( $p$, prime), for example.

\section{Main Definition AND some properties}

Let $G$ be a finite group and let $H$ be a subgroup of $G$. Denote by $G / H$ the set of (left) cosets of $H$ in $G$. The natural left action of $G$ on the set $G / H$ extends linearly to the complex vector space having this set as basis, which we denote by $\mathbf{C}(G / H)$.

Let $\operatorname{Irr}(G)$ denote the set of irreducible characters of $G$. Let $\chi \in \operatorname{Irr}(G)$. Define a form $B_{H}^{\chi}$ on $\mathbf{C}(G / H)$ by putting

$$
B_{H}^{\chi}(a H, b H)=\frac{\chi(e)}{|H|} \sum_{h \in H} \chi\left(a^{-1} b h\right)
$$

1991 Mathematics Subject Classification. Primary 20C15 Secondary 20C30, 20B20.

Typeset by $\mathcal{A} \mathcal{M} \mathcal{S}-\mathrm{TEX}_{\mathrm{E}}$ 
(where $e$ is the identity element of $G$ ) and extending linearly in the first component and antilinearly in the second.

1.1 Proposition. $B_{H}^{\chi}$ is a well-defined G-invariant Hermitian form.

Proof. First note that since $\chi$ is conjugation invariant [I, (2.3), p. 14], we have $\chi(g a)=\chi\left(g^{-1} g a g\right)=$ $\chi(a g)$ for all $a, g \in G$.

Suppose $a_{1} H=a H$ and $b_{1} H=b H$ so that $a_{1}=a x$ and $b_{1}=b y$ for some $x, y \in H$. Then for each $h \in H$,

$$
\chi\left(a_{1}^{-1} b_{1} h\right)=\chi\left(x^{-1} a^{-1} b y h\right)=\chi\left(a^{-1} b y h x^{-1}\right) .
$$

As $h$ ranges through $H$, yhx $x^{-1}$ also ranges through $H$, so $B_{H}^{\chi}\left(a_{1} H, b_{1} H\right)=B_{H}^{\chi}(a H, b H)$ and $B_{H}^{\chi}$ is well-defined.

To say that $B_{H}^{\chi}$ is $G$-invariant is to say that $B_{H}^{\chi}(c a H, c b H)=B_{H}^{\chi}(a H, b H)$ for all $a, b, c \in G$ and this is clear.

Finally,

$$
\begin{aligned}
B_{H}^{\chi}(b H, a H) & =\frac{\chi(e)}{|H|} \sum_{h \in H} \chi\left(b^{-1} a h\right)=\frac{\chi(e)}{|H|} \sum_{h \in H} \overline{\chi\left(h^{-1} a^{-1} b\right)} \\
& =\frac{\chi(e)}{|H|} \sum_{h \in H} \overline{\chi\left(a^{-1} b h^{-1}\right)}=\overline{B_{H}^{\chi}(a H, b H)}
\end{aligned}
$$

$(a, b \in G)$, where we have used that $\overline{\chi(g)}=\chi\left(g^{-1}\right)$ for $g \in G\left[\mathrm{I},(2.15)\right.$, p. 20]. Therefore, $B_{H}^{\chi}$ is Hermitian.

Put $\mathcal{C}_{H}^{\chi}:=\mathbf{C}(G / H) / \operatorname{ker} B_{H}^{\chi}$, where $\operatorname{ker} B_{H}^{\chi}:=\left\{x \in \mathbf{C}(G / H): B_{H}^{\chi}(x, y)=0\right.$ for all $y \in$ $\mathbf{C}(G / H)\}$. Then $B_{H}^{\chi}$ induces a well-defined form $\bar{B}_{H}^{\chi}$ on $\mathcal{C}_{H}^{\chi}$ given by $\bar{B}_{H}^{\chi}(\bar{x}, \bar{y})=B_{H}^{\chi}(x, y)(x, y \in$ $\mathbf{C}(G / H)$ ), where here and below we use $\bar{x}$ to denote the coset $x+\operatorname{ker} B_{H}^{\chi}$ (context should keep any confusion from arising over this notation and the usual notation for complex conjugate which we also use). By 1.1, $\operatorname{ker} B_{H}^{\chi}$ is closed under the action of $G$ and so we have a well-defined action of $G$ on $\mathcal{C}_{H}^{\chi}$. Clearly, $\bar{B}_{H}^{\chi}$ is $G$-invariant.

For characters $\psi$ and $\varphi$ of $G$, one defines $(\psi, \varphi)_{H}=\frac{1}{|H|} \sum_{h \in H} \psi(h) \overline{\varphi(h)}[\mathrm{I},(2.16)$, p. 20]. We denote the principal character of $G$ by 1 ( so $1(g)=1$ for all $g \in G$ ).

\subsection{Theorem.}

(1) $\operatorname{dim}_{\mathbf{C}} \mathcal{C}_{H}^{\chi}=\chi(e)(\chi, 1)_{H}$.

(2) The form $\bar{B}_{H}^{\chi}$ is positive definite.

Proof. Let $a_{1} H, \ldots, a_{n} H$ be the distinct left cosets of $H$ in $G$. Then $\left\{a_{i} H: 1 \leq i \leq n\right\}$ is a basis for $\mathbf{C}(G / H)$. Let $A$ be the $n \times n$-matrix with $(i, j)$-entry $B_{H}^{\chi}\left(a_{i} H, a_{j} H\right) /|G: H|$, where $|G: H|$ is the index of $H$ in $G$. (So $A$ is $|G: H|^{-1}$ times the matrix of the form $B_{H}^{\chi}$ relative to the above basis.) We claim that $A^{2}=A$. The $(i, j)$-entry of $A^{2}$ is

$$
\begin{aligned}
|G: H|^{-2} \sum_{k=1}^{n} B_{H}^{\chi}\left(a_{i} H, a_{k} H\right) B_{h}^{\chi}\left(a_{k} H, a_{j} H\right) & =\frac{\chi(e)^{2}}{|G|^{2}} \sum_{k=1}^{n}\left(\sum_{h \in H} \chi\left(a_{i}^{-1} a_{k} h\right)\right)\left(\sum_{l \in H} \chi\left(a_{k}^{-1} a_{j} l\right)\right) \\
& =\frac{\chi(e)^{2}}{|G|^{2}} \sum_{l \in H} \sum_{\substack{h \in H \\
1 \leq k \leq n}} \chi\left(a_{k} h a_{i}^{-1}\right) \chi\left(a_{j} l h^{-1} a_{k}^{-1}\right) .
\end{aligned}
$$


Replacing $a_{i} h^{-1} a_{k}^{-1}$ with $g$ we have $\chi\left(a_{j} l h^{-1} a_{k}^{-1}\right)=\chi\left(a_{j} l a_{i}^{-1} g\right)=\chi\left(g a_{j} l a_{i}^{-1}\right)$, so the expression above becomes

$$
\frac{\chi(e)^{2}}{|G|^{2}} \sum_{l \in H} \sum_{g \in G} \chi\left(g^{-1}\right) \chi\left(g a_{j} l a_{i}^{-1}\right)=\frac{\chi(e)}{|G|} \sum_{l \in H} \chi\left(a_{j} l a_{i}^{-1}\right)
$$

using the Generalized Orthogonality Relation [I, p. 19, (2.13)]. Since $\chi\left(a_{j} l a_{i}^{-1}\right)=\chi\left(a_{i}^{-1} a_{j} l\right)$, this last expression is $B_{H}^{\chi}\left(a_{i} H, a_{j} H\right) /|G: H|$, which is the $(i, j)$-entry of $A$. Thus, $A^{2}=A$ as claimed.

Now $A$ is Hermitian by 1.1 , so it is similar to a diagonal matrix with the eigenvalues of $A$ along the main diagonal. But since $A^{2}=A$, an eigenvalue of $A$ is either 1 or 0 . Hence, the rank of $A$ is equal to the trace of $A$. But

$$
\operatorname{tr} A=\frac{1}{|G: H|} \sum_{i=1}^{n} B_{H}^{\chi}\left(a_{i} H, a_{i} H\right)=\frac{\chi(e)}{|G|} \sum_{i=1}^{n} \sum_{h \in H} \chi(h)=\frac{\chi(e)}{|H|} \sum_{h \in H} \chi(h)=\chi(e)(\chi, 1)_{H} .
$$

Since $\operatorname{dim}_{\mathbf{C}} \mathcal{C}_{H}^{\chi}=\operatorname{rank} A,(1)$ follows.

Finally, by the preceding paragraph, the form $B_{H}^{\chi}$ on $\mathbf{C}(G / H)$ is positive semidefinite, so that the induced form $\bar{B}_{H}^{\chi}$ on $\mathcal{C}_{H}^{\chi}$ is positive definite. This proves (2).

We shall call $G$ an o-basis group if for every $H \leq G$ and $\chi \in \operatorname{Irr}(G)$ the vector space $\mathcal{C}_{H}^{\chi}$ has a basis that is orthogonal relative to $\bar{B}_{H}^{\chi}$ consisting entirely of elements of the form $\overline{a H}(a \in G)$. Such a basis shall be called an o-basis of $\mathcal{C}_{H}^{\chi}$.

1.3 Corollary. The following are equivalent:

(1) $G$ is an o-basis group.

(2) For each $H \leq G$ and each $\chi \in \operatorname{Irr}(G)$, there exist at least $\chi(e)(\chi, 1)_{H}$ cosets of $H$ in $G$ that are mutually orthogonal relative to $B_{H}^{\chi}$.

(3) For each $H \leq G$ and each nonlinear $\chi \in \operatorname{Irr}(G)$ with $(\chi, 1)_{H} \neq 0$, there exist at least $\chi(e)(\chi, 1)_{H}$ cosets of $H$ in $G$ that are mutually orthogonal relative to $B_{H}^{\chi}$.

Proof. We first observe that for every $a, b \in G$,

$$
\bar{B}_{H}^{\chi}(\overline{a H}, \overline{b H})=B_{H}^{\chi}(a H, b H)
$$

so that $\overline{a H}$ and $\overline{b H}$ are orthogonal relative to $\bar{B}_{H}^{\chi}$ if and only if $a H$ and $b H$ are orthogonal relative to $B_{H}^{\chi}$.

Assume that $G$ is an o-basis group and let $H \leq G$ and $\chi \in \operatorname{Irr}(G)$. There exists an o-basis $\left\{\overline{a_{1} H}, \ldots, \overline{a_{t} H}\right\}$ (possibly empty with $t=0$ ) of $\mathcal{C}_{H}^{\chi}$. By $1.2, t=\chi(e)(\chi, 1)_{H}$ and, by the above argument, $a_{1} H, \ldots, a_{t} H$ are mutually orthogonal relative to $B_{H}^{\chi}$. This shows that (1) implies (2).

That (2) implies (3) is obvious.

Finally, assume (3) holds. Let $H \leq G$ and $\chi \in \operatorname{Irr}(G)$. If $(\chi, 1)_{H}=0$, then $\operatorname{dim} \mathcal{C}_{H}^{\chi}=0$, so the empty set is an o-basis of $\mathcal{C}_{H}^{\chi}$. Assume that $(\chi, 1)_{H} \neq 0$. Note that, in particular,

$$
\bar{B}_{H}^{\chi}(\overline{a H}, \overline{a H})=\frac{\chi(e)}{|H|} \sum_{h \in H} \chi(h)=\chi(e)(\chi, 1)_{H} \neq 0
$$

so that $\overline{a H} \neq 0$ for all $a \in G$. If $\chi$ is linear, then $\operatorname{dim} \mathcal{C}_{H}^{\chi}=1$, so $\{\bar{H}\}$ is an o-basis of $\mathcal{C}_{H}^{\chi}$. Assume that $\chi$ is nonlinear. By assumption, there exist $t=\chi(e)(\chi, 1)_{H}$ cosets $a_{1} H, \ldots, a_{t} H$ that are mutually orthogonal relative to $B_{H}^{\chi}$. Then $\left\{\overline{a_{1} H}, \ldots, \overline{a_{t} H}\right\}$ is orthogonal relative to $\bar{B}_{H}^{\chi}$ and, since $\overline{a_{i} H} \neq 0$ for each $i$, this set is linearly independent and hence an o-basis of $\mathcal{C}_{H}^{\chi}$ (using 1.2).

Remarks. (1) Since the irreducible characters of an abelian group are all linear, it follows vacuously from condition (3) of 1.3 that every abelian group is an o-basis group.

(2) In view of 1.3, the proof of Theorem 3.1 in [HT] shows that if $G$ is a dihedral group of order $2^{k}(k \geq 0)$, then $G$ is an o-basis group. (See also [HT, Remark 2, p. 27].) We shall recover this result as a special case of 3.1 below. 
1.4 Proposition. Let $G$ be an o-basis group. For each $H \leq G$ and $\chi \in \operatorname{Irr}(G)$ there exist at least $\chi(e)(\chi, 1)_{H}-1$ cosets $a H$ for which $\sum_{h \in H} \chi(a h)=0$. In particular, each $\chi \in \operatorname{Irr}(G)$ has at least $\chi(e)^{2}-1$ zeros.

Proof. Let $H \leq G$ and $\chi \in \operatorname{Irr}(G)$. If $(\chi, 1)_{H}=0$, then the claim is vacuously satisfied, so assume $(\chi, 1)_{H} \neq 0$. By assumption and 1.3 there exist $t=\chi(e)(\chi, 1)_{H}$ cosets $a_{1} H, \ldots, a_{t} H$ that are mutually orthogonal relative to $B_{H}^{\chi}$. By the $G$-invariance of $B_{H}^{\chi}(1.1)$, we may assume that $a_{1}=e$. For each $1<i \leq t$ we have

$$
0=B_{H}^{\chi}\left(a_{1} H, a_{i} H\right)=\frac{\chi(e)}{|H|} \sum_{h \in H} \chi\left(a_{i} h\right)
$$

and this proves the first statement. The second statement follows by letting $H=\{e\}$.

In the remainder of this section, we study the o-basis group property as it relates to homomorphic images.

Let $N \triangleleft G$, let $\chi \in \operatorname{Irr}(G)$ and assume that $N \subseteq \operatorname{ker} \chi$. Put $\hat{G}:=G / N$ and denote by $\hat{a}$ the image of $a \in G$ under the canonical epimorphism $G \rightarrow \hat{G}$. The function $\hat{\chi}: \hat{G} \rightarrow \mathbf{C}$ given by $\hat{\chi}(\hat{a})=\chi(a)$ is a well-defined irreducible character of $\hat{G}$ [I, (2.22), p. 24]. Let $H$ be a subgroup of $G$.

1.5 Proposition. Let the notation be as above. The linear map $\varphi: \mathcal{C}_{H}^{\chi} \rightarrow \mathcal{C}_{\hat{H}}^{\hat{\chi}}$ given by $\varphi(\overline{a H})=\overline{\hat{a} \hat{H}}$ is a well-defined linear isometry. In particular, $\mathcal{C}_{H}^{\chi}$ has an o-basis if and only if $\mathcal{C}_{\hat{H}}^{\hat{\chi}}$ has an o-basis.

Proof. Put $I=H \cap N$ and let $h_{1} I, \ldots, h_{n} I$ be the distinct elements of $H / I$. By an isomorphism theorem, $\hat{H} \cong H / I$ and $\hat{h}_{1}, \ldots, \hat{h}_{n}$ are the distinct elements of $\hat{H}$.

Let $a, b \in G$. Using that $\chi$ is constant on each coset of $I$, we get

$$
\begin{aligned}
B_{H}^{\chi}(a H, b H) & =\frac{\chi(e)}{|H|} \sum_{h \in H} \chi\left(a^{-1} b h\right)=\frac{\chi(e)}{|H: I|} \sum_{i=1}^{n} \chi\left(a^{-1} b h_{i}\right) \\
& =\frac{\hat{\chi}(\hat{e})}{|\hat{H}|} \sum_{i=1}^{n} \hat{\chi}\left(\hat{a}^{-1} \hat{b} \hat{h}_{i}\right)=B_{\hat{H}}^{\hat{\chi}}(\hat{a} \hat{H}, \hat{b} \hat{H}) .
\end{aligned}
$$

In particular, the linear map $\mathbf{C}(G / H) \rightarrow \mathbf{C}(\hat{G} / \hat{H})$ given by $a H \mapsto \hat{a} \hat{H}$ sends the kernel of $B_{H}^{\chi}$ into the kernel of $B_{\hat{H}}^{\hat{\chi}}$ so that $\varphi$ is well-defined. Clearly $\varphi$ is surjective. Finally, if $x \in \operatorname{ker} \varphi$, then

$$
\bar{B}_{H}^{\chi}(x, x)=\bar{B}_{\hat{H}}^{\hat{\chi}}(\varphi(x), \varphi(x))=0,
$$

so that $x=0$ since $\bar{B}_{H}^{\chi}$ is definite (1.2). It follows that $\varphi$ is injective.

1.6 Corollary. The class of o-basis groups is closed under taking homomorphic images.

Proof. Let $G$ be an o-basis group and let $N$ be a normal subgroup of $G$. By the First Isomorphism Theorem it suffices to show that $\hat{G}:=G / N$ is an o-basis group.

Let $\hat{H} \leq \hat{G}$ and let $\hat{\chi} \in \operatorname{Irr}(\hat{G})$. With $\chi: G \rightarrow \mathbf{C}$ defined by $\chi(a)=\hat{\chi}(a N)$, we have $\chi \in \operatorname{Irr}(G)$ and $N \subseteq \operatorname{ker} \chi$. Also, $\hat{H}=H / N$ for some $H \leq G$ (with $H \supseteq N$ ). By assumption, $\mathcal{C}_{H}^{\chi}$ has an o-basis, so $\mathcal{C}_{\hat{H}}^{\hat{\chi}}$ has an o-basis as well by 1.5 . 


\section{Orthogonal BASES OF SYMMETRIZED TENSOR SPACES}

In this section, we study connections between the notion of an o-basis group and the existence of special bases (called o-bases) of symmetrized tensor spaces.

Fix positive integers $m$ and $n$ and put $\Gamma_{n, m}=\left\{\gamma \in \mathbf{Z}^{n}: 1 \leq \gamma_{i} \leq m\right\}$. Let $G$ be a subgroup of the symmetric group $S_{n}$. There is a right action of $G$ on $\Gamma_{n, m}$ given by $\gamma \sigma=\left(\gamma_{\sigma(1)}, \ldots, \gamma_{\sigma(n)}\right)$ $\left(\gamma \in \Gamma_{n, m}, \sigma \in G\right)$.

Let $V$ be a complex inner product space of dimension $m$ and let $\left\{e_{1}, \ldots, e_{m}\right\}$ be an orthonormal basis of $V$. To avoid trivialities, we assume that $m \geq 2$. Denote by $V^{\otimes n}$ the $n$-fold tensor power of $V$. For $\gamma \in \Gamma_{n, m}$, put $e_{\gamma}:=e_{\gamma_{1}} \otimes \cdots \otimes e_{\gamma_{n}} \in V^{\otimes n}$. Then $\left\{e_{\gamma}: \gamma \in \Gamma_{n, m}\right\}$ is a basis for $V^{\otimes n}$.

Let $\chi \in \operatorname{Irr}(G)$. The symmetrizer relative to $\chi$ is the element of the group algebra $\mathbf{C} G$ of $G$ given by $s^{\chi}:=\frac{\chi(e)}{|G|} \sum_{\sigma \in G} \chi(\sigma) \sigma$. For $\gamma \in \Gamma_{n, m}$, put $e_{\gamma}^{\chi}:=s^{\chi} e_{\gamma}$, where we view $V^{\otimes n}$ as a left $\mathbf{C} G$-module via $\sigma e_{\gamma}=e_{\gamma \sigma^{-1}}(\sigma \in G)$. We shall refer to $e_{\gamma}^{\chi}$ as a standard symmetrized tensor (some authors use the term decomposable tensor).

The inner product on $V$ induces an inner product on $V^{\otimes n}$. If $W$ is a subspace of $V^{\otimes n}$ then an orthogonal basis of $W$ consisting entirely of standard symmetrized tensors shall be called an o-basis of $W$ (relative to $G$ ).

Choose a set $\triangle$ of representatives of the orbits of $\Gamma_{n, m}$ under the right action of $G$ given above. Then $V^{\otimes n}=\bigoplus V_{\gamma}^{\chi}$ (orthogonal direct sum), where $V_{\gamma}^{\chi}:=\left\langle e_{\gamma \sigma}^{\chi}: \sigma \in G\right\rangle$ and the sum is over all $\chi \in \operatorname{Irr}(G), \gamma \in \triangle$ (cf. [F], [M]).

2.1 Theorem. If $G$ is an o-basis group and $\varphi: G \rightarrow S_{n}(n \in \mathbf{N})$ is a homomorphism, then $V^{\otimes n}$ has an o-basis relative to $\varphi(G)$.

Proof. Let $G$ be an o-basis group and let $\varphi: G \rightarrow S_{n}(n \in \mathbf{N})$ be a homomorphism. Put $J=\varphi(G)$ and fix $\psi \in \operatorname{Irr}(J)$ and $\gamma \in \Gamma_{n, m}$. It is enough to show that $V_{\gamma}^{\psi}$ has an o-basis (relative to $J$ ). Set $H=\varphi^{-1}\left(J_{\gamma}\right)$, where $J_{\gamma}$ is the stabilizer of $\gamma$ under the right action of $J$ on $\Gamma_{n, m}$. Also, put $\chi=\psi \circ \varphi \in \operatorname{Irr}(G)$. By the definition of o-basis group, $\mathcal{C}_{H}^{\chi}$ has an o-basis $\left\{\overline{a_{1} H}, \ldots, \overline{a_{t} H}\right\}$ and by $1.2, t=\chi(e)(\chi, 1)_{H}$. Put $\theta_{i}=\varphi\left(a_{i}\right)^{-1}$. We claim that $\left\{e_{\gamma \theta_{i}}^{\psi}: 1 \leq i \leq t\right\}$ is an o-basis of $V_{\gamma}^{\psi}$. For $1 \leq i, j \leq t$, we have

$$
\bar{B}_{H}^{\chi}\left(\overline{a_{i} H}, \overline{a_{j} H}\right)=\frac{\chi(e)}{|H|} \sum_{h \in H} \chi\left(a_{i}^{-1} a_{j} h\right)=\frac{\psi(e)}{\left|J_{\gamma}\right|} \sum_{\sigma \in J_{\gamma}} \psi\left(\theta_{i} \theta_{j}^{-1} \sigma\right)=\left(e_{\gamma \theta_{i} \theta_{j}^{-1}}^{\psi}, e_{\gamma}^{\psi}\right)=\left(e_{\gamma \theta_{i}}^{\psi}, e_{\gamma \theta_{j}}^{\psi}\right),
$$

where the next to the last equality is from [F, p. 339]. The equation above with $j=i$ shows that each $e_{\gamma \theta_{i}}^{\psi}$ is nonzero (using definiteness of $\bar{B}_{H}^{\chi}(1.2)$ ). On the other hand, the equation above with $j \neq i$ shows that the vectors $e_{\gamma \theta_{i}}^{\psi}$ are mutually orthogonal. In particular, the set $\left\{e_{\gamma \theta_{i}}^{\psi}: 1 \leq i \leq t\right\}$ is linearly independent. Also, by [F, p. 339],

$$
\operatorname{dim}_{\mathbf{C}} V_{\gamma}^{\psi}=\frac{\psi(e)}{\left|J_{\gamma}\right|} \sum_{\sigma \in J_{\gamma}} \psi(\sigma)=\frac{\chi(e)}{|H|} \sum_{h \in H} \chi(h)=\chi(e)(\chi, 1)_{H}=t
$$

so the theorem follows.

2.2 Corollary. The following groups are not o-basis groups:

(1) any dihedral group $D_{n}$ (of order $2 n$ ) with $n$ not a power of 2 ,

(2) any 2-transitive subgroup of $S_{n}$ with $n \geq 3$ (e.g., the alternating group $A_{n}, n \geq 4$ and the symmetric group $S_{n}, n \geq 3$ ),

(3) any finite simple group of Lie type. 
Proof. Let $G$ be one of the groups in the list above. In view of 2.1, it is enough to find a homomorphism $\varphi: G \rightarrow S_{n}$ for some $n$ such that $V^{\otimes n}$ does not have an o-basis relative to $\varphi(G)$.

Case (1) is given in [HT, Corollary 3.3, p. 27] with $\varphi: D_{n} \rightarrow S_{n}$ the natural embedding, case (2) is given in [H, Theorem, p. 242] with $\varphi: G \rightarrow S_{n}$ the inclusion map, and case (3) is given in [A, Theorem 5.1, p. 428] with $\varphi: G \rightarrow S_{n}$ the embedding induced by the natural action of $G$ on $G / B$, where $B$ is a Borel subgroup and $n=|G: B|$.

The converse of 2.1 does not hold in general since it is possible to have a group homomorphism $\varphi: G \rightarrow S_{n}$ such that $V^{\otimes n}$ has an o-basis relative to $\varphi(G)$ with $G$ not an o-basis group. (Indeed, one can let $G$ be any of the groups in 2.2 and let $\varphi: G \rightarrow S_{n}$ (any $n$ ) be the trivial homomorphism. Then the identity map on $V^{\otimes n}$ is the sole symmetrizer and $\left\{e_{\gamma}: \gamma \in \Gamma_{n, m}\right\}$ is an o-basis of $V^{\otimes n}$ relative to $\varphi(G)=\{e\}$.) However, the next theorem provides a characterization of o-basis group expressed in terms of symmetrized tensors. In its statement, the Cayley embedding $\varphi: G \rightarrow S_{n}$ is the homomorphism that takes $g \in G$ to the permutation $\varphi(g)$ on $G$ given by $\varphi(g)(h)=g h(h \in G)$, this permutation being viewed as an element of $S_{n}$, where $n=|G|$.

2.3 Theorem. Let $G$ be a finite group, let $n=|G|$, and let $\varphi: G \rightarrow S_{n}$ be the Cayley embedding. Then $G$ is an o-basis group if and only if $V^{\otimes n}$ has an o-basis relative to $\varphi(G)$.

Proof. One implication follows from 2.1. Now assume that $V^{\otimes n}$ has an o-basis relative to $\varphi(G)$. Fix $H \leq G$ and $\chi \in \operatorname{Irr}(G)$. We view $\Gamma_{n, m}$ as the set of functions from $G$ to $\{1, \ldots, m\}$ using the same one-to-one correspondence $G \rightarrow\{1, \ldots, n\}$ by which we identify the symmetric group on $G$ with $S_{n}$. Define $\gamma \in \Gamma_{n, m}$ by

$$
\gamma_{g}= \begin{cases}1, & \text { if } g \in H, \\ 2, & \text { if } g \notin H .\end{cases}
$$

Then clearly the stabilizer of $\gamma$ in $G$ is $H$. Put $\psi=\left.\chi \circ \varphi^{-1}\right|_{\varphi(G)} \in \operatorname{Irr}(\varphi(G)$ ). By assumption (and the orthogonal direct sum decomposition given before 2.1), $V_{\gamma}^{\psi}$ has an o-basis, that is, there exist $g_{1}, \ldots, g_{t} \in G$ with $t=\operatorname{dim}_{\mathbf{C}} V_{\gamma}^{\psi}$ such that $\left\{e_{\gamma \varphi\left(g_{i}\right)}^{\psi}: 1 \leq i \leq t\right\}$ is an orthogonal basis of $V_{\gamma}^{\chi}$. The computations in the proof of 2.1 show that for $1 \leq i, j \leq t, \bar{B}_{H}^{\chi}\left(\overline{g_{i}^{-1} H}, \overline{g_{j}^{-1} H}\right)=\left(e_{\gamma \varphi\left(g_{i}\right)}^{\psi}, e_{\gamma \varphi\left(g_{j}\right)}^{\psi}\right)$ and that $\operatorname{dim}_{\mathbf{C}} V_{\gamma}^{\psi}=\chi(e)(\chi, 1)_{H}$, so, arguing as in that same proof and in view of $1.2\left\{\overline{g_{i}^{-1} H}\right.$ : $1 \leq i \leq t\}$ is an o-basis of $\mathcal{C}_{H}^{\chi}$.

\section{A sufficient CONDITION AND EXAMPLES}

In the first theorem of this section we consider a certain class of $p$-groups and show that its members are o-basis groups. This theorem is used in 3.2 to provide a list of familiar groups that are o-basis groups.

3.1 Theorem. Let $G$ be a finite p-group ( $p$, prime) and assume that $G$ has an abelian normal subgroup $A$ and a cyclic normal subgroup $C$ with $C \subseteq A$ satisfying $|G: A| \leq p$ and $|A: C| \leq p$. Then $G$ is an o-basis group.

Proof. We verify the characterization of o-basis group given in 1.3. Let $H \leq G$ and $\chi \in \operatorname{Irr}(G)$ with $\chi$ nonlinear and $(\chi, 1)_{H} \neq 0$. Note that since $\chi$ is nonlinear, $G$ is nonabelian so that $|G|>p^{2}$ and $C \neq\{e\}$. A quotient of $G$ clearly satisfies the hypotheses of the theorem so we assume, without loss of generality in view of 1.5 , that $\chi$ is faithful.

We claim that $H \cap C=\{e\}$. Let $J=H \cap C$. Now $J$ is a characteristic subgroup of $C$ (as is any subgroup of $C$ since $C$ is cyclic) and $C$ is a normal subgroup of $G$. Hence, $J$ is a normal subgroup of $G$. Since $(\chi, 1)_{H} \neq 0$, it follows that $(\chi, 1)_{J} \neq 0$. Then [I, (6.7), p. 81] says that $J \subseteq \operatorname{ker} \chi=\{e\}$. Thus $H \cap C=\{e\}$, as claimed. 
We now claim that $|H|$ is either 1 or $p$. Since $H \cap C=\{e\}$, we have $|H||C|=|H C| \leq|G|$. Now $|C|=|G| /|G: C| \geq|G| / p^{2}$, so $|H| \leq p^{2}$. Moreover, $|H|$ divides $|G|$ which is a power of $p$, so $|H| \in\left\{1, p, p^{2}\right\}$. Suppose that $|H|=p^{2}$. Then $|G: A|=p=|A: C|$. In particular, $|H \cap A|=p$ so that $H \cap A=\langle h\rangle$ for some $h \in H$. Moreover, $H \nsubseteq \subseteq A$ so there exists some $x \in H-A$. Then clearly $G=\langle C, h, x\rangle$. Now $H$ is abelian since it has order $p^{2}$, so it follows that $h$ is in the center $Z(G)$ of $G$. Now $G$ acts by conjugation on $C$ and hence fixes a nonidentity element $c$ of $C$ [Hu, Lemma 5.1, p. 93]. Thus $Z(G)$ contains $\langle c\rangle \times\langle h\rangle$. But this contradicts that $Z(G)$ is cyclic since $\chi$ is faithful [I, (2.32), p. 29]. We conclude that $|H|$ is either 1 or $p$, as claimed.

By Ito's Theorem [I, (6.15), p. 84], $\chi(e)$ divides $|G: A|$ which is either 1 or $p$. We are assuming that $\chi$ is nonlinear, so we have $\chi(e)=p$ and $|G: A|=p$. Let $\lambda$ be an irreducible constituent of $\chi_{A}$. Since $A$ is abelian, we have $\lambda(e)=1$. Frobenius Reciprocity gives $\left(\chi, \lambda^{G}\right)_{G}=(\chi, \lambda)_{A} \geq 1$. Since $\chi$ and $\lambda^{G}$ both have degree $p$, we conclude that $\chi=\lambda^{G}$.

For later use, we observe that $\lambda_{C}$ is faithful. Indeed, $\operatorname{ker} \lambda_{C}$ is a characteristic subgroup of $C$ and hence a normal subgroup of $G$ so that

$$
\operatorname{ker} \lambda_{C}=\bigcap_{x \in G}\left(\operatorname{ker} \lambda_{C}\right)^{x} \subseteq \bigcap_{x \in G}(\operatorname{ker} \lambda)^{x}=\operatorname{ker} \lambda^{G}=\operatorname{ker} \chi=\{e\}
$$

using [I, (5.11), p. 67] and the fact that $\chi$ is faithful.

Let $N=C \cap Z(G)$. Assume for the moment that $N \neq C$. The conjugation action of $G$ on $C$ induces a well-defined action of $G$ on $C / N$ given by $(c N)^{x}=c^{x} N$ for $c \in C, x \in G$. According to $[\mathrm{Hu}$, Theorem 5.2, p. 93], this action fixes the elements of a subgroup of order $p$, which can be expressed in the form $D / N$ with $D$ a subgroup of $C$ containing $N$.

So far, $D$ is defined if $N \neq C$. If $N=C$, put $D=A$ and note that $C \neq A$, for otherwise, $N=A$ and the inertia subgroup of $\lambda$ is $G$, contradicting that $\lambda^{G}=\chi$ is irreducible by [I, (6.1), p. 95]. We have that $D$ is a normal subgroup of $G$ and $|D: N|=p$.

We claim that $\chi_{D}=\sum_{i=0}^{p-1} \eta_{i}$, with the $\eta_{i}$ distinct linear characters of $D$. If $N=C$, then this follows from [I, (6.19), p. 86], so now assume that $N \neq C$. By Mackey's Theorem [I, (5.6), p. 74], $\chi_{D}=\sum_{i=0}^{p-1} \lambda_{D}^{x^{i}}$, where $G / A=\langle x A\rangle$. Let $0 \leq i \leq j<p$ and assume that $\lambda_{D}^{x^{i}}=\lambda_{D}^{x^{j}}$. It suffices to show that $i=j$. We have $D / N=\langle d N\rangle$ for some $d \in D-N$. Then $\lambda\left(x^{x^{i}} d\right)=\lambda^{x^{i}}(d)=\lambda^{x^{j}}(d)=$ $\lambda\left({ }^{x^{j}} d\right)$, which implies that ${ }^{x^{i}} d={ }^{x^{j}} d$ since $\lambda_{C}$ is faithful. Therefore, ${ }^{x^{j-i}} d=d$. If $i \neq j$, then $0<j-i<p$, so $G=\left\langle x^{j-i}, A\right\rangle$ and it follows that $d \in Z(G) \cap C=N$, a contradiction. Thus, $i=j$. Our claim follows by putting $\eta_{i}:=\lambda_{D}^{x^{i}}(0 \leq i<p)$.

Next, we show that $\chi$ vanishes on $D-N$. By Clifford's Theorem [I, (6.2), p. 79], we have $\chi_{N}=p \mu$ for some linear character $\mu$ of $N$. With the notation as in the preceding paragraph we have

$$
\sum_{i=0}^{p-1}\left(\mu, \eta_{i}\right)_{N}=(\mu, \chi)_{N}=p
$$

and, since each $\left(\mu, \eta_{i}\right)_{N}$ is at most one, it follows that $\left(\mu, \eta_{i}\right)_{N}=1$ for all $i$. Therefore, $\left(\mu^{D}, \eta_{i}\right)_{D}=$ $\left(\mu, \eta_{i}\right)_{N}=1$ for all $i$, where we have used Frobenius Reciprocity. Since $\mu^{D}$ has degree $p$, it follows that $\chi_{D}=\mu^{D}$ and so $\chi$ vanishes on $D-N$ by the definition of the induced character [I, (5.1), p. $62]$ and the normality of $N$.

Define natural numbers $s$ and $t$ as follows:

$$
(s, t)= \begin{cases}(p, p), & \text { if }|H|=1, \\ (1, p), & \text { if }|H|=p, H \subseteq A, \\ (p, 1), & \text { if }|H|=p, H \nsubseteq A .\end{cases}
$$


Since $|H|$ is either 1 or $p$, as observed earlier, this defines $s$ and $t$ in all cases.

As above, we have $G / A=\langle x A\rangle$ and $D / N=\langle d N\rangle$ for some $x \in G$ and $d \in D$. We shall show that the cosets $d^{i} x^{j} H(0 \leq i<s, 0 \leq j<t)$ are mutually orthogonal with respect to $B_{H}^{\chi}$. Let $0 \leq i, k<s$ and $0 \leq j, l<t$ and assume that $(i, j) \neq(k, l)$. First suppose that $j \neq l$. Then $t \neq 1$, so $H \subseteq A$. Therefore, for each $h \in H$, we have $x^{-j} d^{k-i} x^{l} h=x^{l-j} x^{-l} d^{k-i} x^{l} h \in x^{l-j} A \subseteq G-A$. Now $\chi=\lambda^{G}$ and $A$ is a normal subgroup of $G$, so by the definition of the induced character, $\chi$ vanishes on $G-A$, so

$$
B_{H}^{\chi}\left(d^{i} x^{j} H, d^{k} x^{l} H\right)=\frac{\chi(e)}{|H|} \sum_{h \in H} \chi\left(x^{-j} d^{k-i} x^{l} h\right)=0 .
$$

Now suppose that $j=l$. Then $i \neq k$. In particular, $s \neq 1$, so that $H \cap A=\{e\}$. It follows that $x^{-j} d^{k-i} x^{j} h \in G-A$ for $h \in H-\{e\}$, while $x^{-j} d^{k-i} x^{j} \in D-N$. We have noted that $\chi$ vanishes on $G-A$ and on $D-N$, so $\left(^{*}\right)$ is valid in this case as well.

Now we show that $\chi(e)(\chi, 1)_{H}=$ st. If $|H|=1$, then both sides of this equation equal $p^{2}$. Now assume that $|H| \neq 1$, so that $|H|=p$.

We claim that $\chi(h)=0$ for all $e \neq h \in H$. If $H \nsubseteq A$, then $H \cap A=\{e\}$ and the claim follows since $\chi$ vanishes on $G-A$. Now assume that $H \subseteq A$. The socle of $A$ is $Z H$ where $Z$ is the subgroup of $C$ of order $p$ (recalling that $C \neq\{e\}$ by the first paragraph of the proof). Now as the socle, $Z H$ is characteristic in $A$ and hence normal in $G$. As above, we have $G / A=\langle x A\rangle$ for some $x \in G$. The conjugation action of $x$ on $Z H$ fixes the elements of $Z$ and hence induces an action on $Z H / Z$, which must be the trivial action. Writing $H=\langle h\rangle$, we have ${ }^{x} h=z h$ for some $z \in Z$. Moreover, $z \neq e$. (Otherwise, we get $H \triangleleft G$ so that our assumption $(\chi, 1)_{H} \neq 0$ implies that $H \subseteq$ ker $\chi$ [I, (6.7), p. 81] contrary to the fact that $\chi$ is faithful.) By induction, ${ }^{i} h=z^{i} h$ for all $0 \leq i<p$. Therefore, using Mackey's Theorem, we obtain

$$
\chi(h)=\sum_{i=0}^{p-1} \lambda^{x^{i}}(h)=\sum_{i} \lambda\left({ }^{x^{i}} h\right)=\lambda(h) \sum_{i} \lambda(z)^{i}=0,
$$

the last equality due to the fact that $\lambda(z)$ is a primitive $p$ th root of unity (using that $\lambda_{C}$ is faithful as observed above). Since $h$ was an arbitrary generator of $H$, it follows that $\chi(h)=0$ for all $e \neq h \in H$, as claimed.

Finally, according to the previous paragraph, we have

$$
(\chi, 1)_{H}=\frac{1}{|H|} \sum_{h \in H} \chi(h)=\frac{1}{|H|} \chi(e)=1
$$

(still assuming that $|H| \neq 1$ ), so that $\chi(e)(\chi, 1)_{H}=p=s t$, as desired. This completes the proof.

3.2 Corollary. The following groups are o-basis groups (p, prime, $n \geq 1$ ):

(1) any finite abelian group,

(2) the dihedral group $D_{2^{n}}$,

(3) the quaternion group $Q_{2^{n}}$,

(4) the semidihedral group $S_{2^{n}}$,

(5) the group with presentation $\left\langle x, a \mid x^{p}=1=a^{p^{n-1}}, a^{x}=a^{1+p^{n-2}}\right\rangle$,

(6) any group of order $p^{3}$,

(7) any extra-special p-group. 
Proof. Let $G$ be one of the groups in (1-5). According to [R, 5.3.4, p. 136], $G$ is a $p$-group with a cyclic maximal subgroup, say $C$. Therefore, $G$ satisfies the hypotheses of 3.1 with $A=C$.

Next we prove (6). Let $G$ be a group of order $p^{3}$. Then $G$ has a nontrivial center [R, 1.6.14, p. 39] and hence a normal subgroup $C$ of order $p$, which is necessarily cyclic. By the First Sylow Theorem, $G$ has a subgroup $A$ of order $p^{2}$ containing $C$. By reason of order, $A$ is abelian and, again by the First Sylow Theorem, $A$ is normal. The hypotheses of 3.1 are satisfied and therefore $G$ is an o-basis group.

Finally, we prove (7). Let $G$ be an extra-special $p$-group. Then, by definition, $G^{\prime}=Z$ and $|Z|=p$, where $G^{\prime}$ is the commutator subgroup of $G$ and $Z$ is the center of $G$. We shall use the characterization of o-basis group given in 1.3. Let $H \leq G$ and $\chi \in \operatorname{Irr}(G)$ with $\chi$ nonlinear and $(\chi, 1)_{H} \neq 0$. According to [K, Theorems 2.17 and 2.18, pp. 812-813], $|G|=p^{2 r+1}$ for some $r \geq 1$, $\chi(e)=p^{r}, \chi$ is faithful, and $\chi$ vanishes on $G-Z$. Since $\chi$ is faithful, we have $H \cap Z=\{e\}$ (arguing as in the second paragraph of the proof of 3.1). Since $\chi$ vanishes on $G-Z$, we get

$$
(\chi, 1)_{H}=\frac{1}{|H|} \sum_{h \in H} \chi(h)=\frac{\chi(e)}{|H|}=\frac{p^{r}}{|H|} .
$$

Therefore, $t:=\chi(e)(\chi, 1)_{H}=p^{2 r} /|H|=|G: Z H|$. Let $a_{1} Z H, \ldots, a_{t} Z H$ be the distinct cosets of $Z H$ in $G$. Then the cosets $a_{1} H, \ldots, a_{t} H$ are mutually orthogonal relative to $B_{H}^{\chi}$. Indeed, for $i \neq j$ and $h \in H$, we have $a_{i}^{-1} a_{j} h \in G-Z$ so that $\chi\left(a_{i}^{-1} a_{j} h\right)=0$ and therefore $B_{H}^{\chi}\left(a_{i} H, a_{j} H\right)=0$. Thus $G$ is an o-basis group by 1.3 .

Remarks. It was noted earlier that (1) follows trivially from 1.3 and that (2) followed from [HT]. Since a nonabelian group of order $p^{3}$ is extra-special [R, 5.3.8, p. 141], (6) also follows from (7) and $(1)$.

In view of 3.2, one might suspect that every finite $p$-group is an o-basis group. Our final example shows that this is not the case, however.

Example. We exhibit a group of order $3^{4}$ that is not an o-basis group. For each $i \in\{1,2,3,4\}$ let $C_{i}=\left\langle c_{i}\right\rangle$ be a cyclic group of order 3 . There is an action of the group $C_{4}$ on the group $N:=C_{1} \times C_{2} \times C_{3}$ uniquely determined by $c_{1}^{c_{4}}=c_{2}, c_{2}^{c_{4}}=c_{3}, c_{3}^{c_{4}}=c_{1}$, where we view $C_{i} \leq N$ $(i \in\{1,2,3\})$ in the usual way. Let $G$ be the semidirect product $N \rtimes C_{4}$ relative to this action (so in fact $G$ is the wreath product $G=\mathbf{Z}_{3}$ wr $\mathbf{Z}_{3}$ ). Then $|G|=3^{4}$. Let $\lambda$ be the linear character of $N$ satisfying $\lambda\left(c_{1}\right)=\epsilon, \lambda\left(c_{2}\right)=1=\lambda\left(c_{3}\right)$, with $\epsilon^{3}=1, \epsilon \neq 1$, and let $\chi$ be the induced character $\lambda^{G}$. Since $\lambda^{c_{4}^{-1}}\left(c_{1}\right)=\lambda\left(c_{1}^{c_{4}}\right)=\lambda\left(c_{2}\right)=1 \neq \epsilon=\lambda\left(c_{1}\right)$, it follows that the inertia group of $\lambda$ is $N$. Therefore, $\chi$ is irreducible [I, (6.1), p. 95]. By Clifford's theorem [I, (6.2), p. 79], $\chi_{N}=\lambda+\lambda^{c_{4}}+\lambda^{c_{4}^{2}}$. In particular, we have

$$
\chi\left(c_{1}^{i_{1}} c_{2}^{i_{2}} c_{3}^{i_{3}}\right)=\epsilon^{i_{1}}+\epsilon^{i_{2}}+\epsilon^{i_{3}}
$$

$\left(i_{j} \in\{0,1,2\}\right)$. Let $H=C_{3} \leq N$. From the formula above, we get

$$
\frac{1}{|H|} \sum_{h \in H} \chi\left(c_{1}^{i_{1}} c_{2}^{i_{2}} h\right)=\epsilon^{i_{1}}+\epsilon^{i_{2}}
$$

for $i_{j} \in\{0,1,2\}$. We see that this quantitiy is never zero, for if it were, we would have $1=\left(\epsilon^{i_{1}}\right)^{3}=$ $\left(-\epsilon^{i_{2}}\right)^{3}=-1$. It follows that for any $a, b \in N, B_{H}^{\chi}(a H, b H) \neq 0$. Let $a_{1} H, \ldots, a_{t} H$ be a set of mutually orthogonal cosets relative to $B_{H}^{\chi}$. Then these cosets must lie in distinct cosets of $N$ in $G$. Indeed, suppose $a_{i} H, a_{j} H \subseteq a N$ for some $a \in G$ with $i \neq j$. Then using $G$-invariance of $B_{H}^{\chi}(1.1)$, we have $0=B_{H}^{\chi}\left(a_{i} H, a_{j} H\right)=B_{H}^{\chi}\left(a^{-1} a_{i} H, a^{-1} a_{j} H\right)$, which is a contradiction as $a^{-1} a_{i}, a^{-1} a_{j} \in N$. We conclude that $t \leq|G: N|=3$.

On the other hand, the formula above gives $\chi(e)(\chi, 1)_{H}=6$. Therefore, $G$ is not an o-basis group by 1.3 . 


\section{REFERENCES}

[A] D. Alvis, Units in Hecke algebras, J. Algebra 216 no. 2 (1999), 417-430.

$[\mathrm{F}] \quad$ R. Freese, Inequalities for generalized matrix functions based on arbitrary characters, Linear Algebra Appl. 7 (1973), 337-345.

[H] R. R. Holmes, Orthogonal bases of symmetrized tensor spaces, Linear and Multilinear Algebra 39 (1995), 241-243.

[HT] R. R. Holmes and T.-Y. Tam, Symmetry classes of tensors associated with certain groups, Linear and Multilinear Algebra 32 (1992), 21-31.

[Hu] T. W. Hungerford, Algebra, Springer-Verlag, New York, 1974.

[I] I. M. Isaacs, Character Theory of Finite Groups, Academic Press, New York, 1976.

[K] G. Karpilovsky, Group Representations, Volume 1, Part B: Introduction to Group Representations and Characters, North-Holland, Amsterdam, 1992.

[M] R. Merris, Recent advances in symmetry classes of tensors, Linear and Multilinear Algebra 7 (1979), 317-328.

[R] D. J. S. Robinson, A Course in the Theory of Groups, Springer-Verlag, New York, 1982.

[WG] B. Y. Wang and M. P. Gong, A higher symmetry class of tensors with an orthogonal basis of decomposable symmetrized tensors, Linear and Multilinear Algebra 30(1-2) (1991), 61-64.

Department of Mathematics, Auburn University, AL 36849-5310 